\title{
Pertinence of Telehealth in a Rush Conversion to Virtual Allergy Practice During the COVID-19 Outbreak
}

González-Pérez R, Sánchez-Machín I, Poza-Guedes P, Matheu V, Álava-Cruz C, Mederos Luís E

Allergy Department, Hospital Universitario de Canarias, Tenerife, Spain

J Investig Allergol Clin Immunol 2021; Vol. 31(1): 78-80 doi: $10.18176 /$ jiaci.0597

Key words: eHealth. Telemedicine. Allergy practice. Pandemic.

Palabras clave: Salud electrónica. Telemedicina. Práctica alergológica. Pandemia.

Telephone consultation is one of the most basic forms of telehealth [1], which is based on a wide range of information and communication technologies, enabling external companies and institutions to reach a multitude of users and paving the way for data collection and management interventions $[2,3]$. The COVID-19 pandemic has led to an unexpected transition from in-person care to telehealth involving not only primary care, but also medical specialties. In Spain, one of the hardesthit countries in the world for recorded cases of COVID-19, specialist care is provided predominantly by public hospitals via outpatient clinics [4]. We describe our experience facing the challenge of creating a "virtual" allergy department in a tertiary care referral hospital and a peripheral outpatient facility serving a population of 430021 inhabitants in Tenerife, Spain.

This study was approved by the Ethics Committee (CEIC) of Hospital Universitario de Canarias, Tenerife, Spain on February 27, 2020 (reference number ISM-DER-2019-01).

Our daily routine electronic consultations - in use from April 2012 - are text-based, asynchronous, and storeand-forward bidirectionally between a referring clinician and a specialist, thus enabling prioritization of in-person visits according to the medical information provided [5]. Unexpectedly, changes that would typically require months of planning ahead were condensed to go fully virtual in less than 48 hours from Saturday, March 14, when the mandatory national emergency quarantine was declared in Spain. By expeditiously adopting telehealth, the Servicio Canario de Salud, which is the main local stakeholder, granted access through a virtual private network so that specialist medical staff could optimize the number of patients coming in [6]. The number of clinicians who physically staff the office was reduced dramatically to 1 physician and 2 nurses per day, while the remaining staff provided telehealth from their home. Furthermore, in-person care was limited to essential procedures (ie, drug desensitization, administration of biologics, and venom immunotherapy). A specific in-person protective health care circuit was developed (only 1 patient per waiting room) so that patients could receive care with a reduced risk of exposure. 
As time was the main concern, our telehealth practice was almost exclusively based on telephone visits; less than $5 \%$ of patients were reached by WhatsApp, e-mail, and/or our website. In the telephone consultations, clinicians went through scheduled appointments, making triage decisions for face-to-face visits and distinguishing between first-time referred users and those with a second in-patient visit. All telephone medical visits were immediately transcribed by the attending physician to the user's electronic record. Although daily routine procedures have been drastically reduced (ie, cutaneous and pulmonary function tests), the use of protocols including a problem-oriented medical diagnosis according to national guidelines and a corresponding electronic prescription enables in-person visits to be deferred for several weeks, thus lowering the risk of exposure [7]. To physicians' surprise and in only 4 working days, a telehealth approach based on a single medical intervention with patients remaining at home prevented 278 users (91.14\%) from coming in (Figure). Likewise, telephone consultation enabled prompt management of $56.25 \%$ (144 persons) of previously scheduled second visits $(27.7 \%$ of whom had been discharged from the Allergy Department) through clinical follow-up by the consultant. This included interpretation of laboratory test results and the use of the electronic prescription service to send prescriptions and preauthorization reviews before patients could be effectively reimbursed. Moreover, almost $37 \%$ of ordinary consultations on adverse drug reactions were concluded online or scheduled for subsequent in vivo procedures (skin testing and/or drug challenge) within the following 3 months, thus making telehealth the mainstay for the future planning of first consultations for drug allergy in our practice.

The response was generally one of relief and surprise, as patients were wary of coming to the Allergy Department and unaware that telehealth care was available at this stage. Most users expressed their gratitude for being called 48 hours ahead of the confirmed in-person visit to prevent nonessential exposures, while physicians were also satisfied by the extent of care provided remotely.

The reasons for the slow uptake of telehealth are multifaceted and diverse depending on the country and

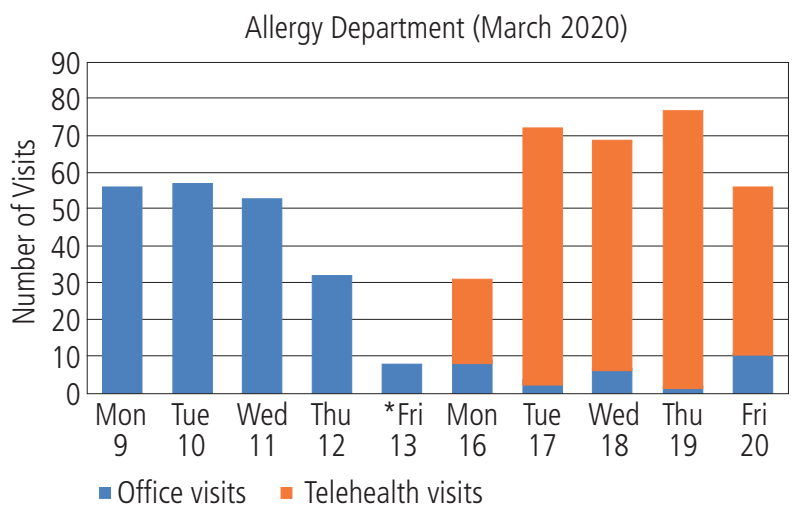

Figure. Number of visits during the transition to telehealth in the allergy service.

*Last working day before the COVID-19 national emergency was declared in Spain. include clinician willingness, financial reimbursement, and reorganization of the health system [8]. Patient barriers including age, socioeconomic status, and level of education are the main limitations we came across, with a proportion of the population not having access to a smartphone and new technology or not reachable because of inaccurate contact details in the corresponding medical records $(1.96 \%$, ie, 6 out of 305 scheduled in-person appointments) [9]. Timeconsuming questions at the start of the virtual consultation ie, asking patients to find a quiet private space to facilitate a telephone consultation, may be overcome by scheduled on-line appointment reminders the day before, thus enabling current workflows to be maintained [10].

In our opinion, appropriate use of telemedicine should become part of the curriculum for the training of health care professionals in order to ensure an adequate level of awareness. In addition, telemedicine is particularly useful for substituting or supplementing face-to-face specialist consultations to cover a wide range of patient needs. It has proven to be a valuable tool for facilitating health care during the mandatory national restricted mobility because of COVID-19. As with other forms of care delivery, telemedicine ought to be personalized.

\section{Acknowledgments}

The authors would like to express their deepest gratitude and admiration to all those health care workers struggling with the COVID-19 pandemic worldwide.

\section{Funding}

The authors declare that no funding was received for the present study.

\section{Conflicts of Interest}

The authors declare that they have no conflicts of interest.

\section{References}

1. https://www.who.int/sustainable-development/health-sector/ strategies/telehealth/en/ (Last accessed April 9, 2020).

2. Alvarez-Perea A, Cabrera-Freitag $P$, Fuentes-Aparicio $V_{\text {, }}$ Infante S, Zapatero L, Zubeldia J. Social media as a tool for the management of food allergy in children. J Investig Allergol Clin Immunol. 2018;28:233-40.

3. Alvarez-Perea A, Sánchez-García S, Muñoz Cano R, AntolínAmérigo D, Tsilochristou O, Stukus DR. Impact Of "eHealth" in Allergic Diseases and Allergic Patients. J Investig Allergol Clin Immunol. 2019;29(2):94-102.

4. https://covid19.who.int (Last accessed June 4, 2020).

5. Sánchez-Machín I, Poza-Guedes P, González Pérez R, Barrios Y, Matheu V. Adapting Waiting-List For Allergy By Health Care On-Line: Coordination Between Providers and Allergist In The Public System. J Allergy Clin Immunol. 2014;133:AB66.

6. https://boe.es/boe/dias/2020/03/14/ (Last accessed April 8, 2020).

7. https://www.gemasma.com (Last accessed June 2, 2020). 
8. Smith $A C$, Emma E, Snoswell CE, Haydon $H$, Clemensen J, Caffery LJ. Telehealth for global emergencies: Implications for coronavirus disease 2019 (COVID-19). J Telemed Telecare. 2020;0:1-5.

9. Sørensen K, Pelikan JM, Röthlin F, Ganahl K, Slonska Z, Doyle $\mathrm{G}$, et al. Health literacy in Europe: comparative results of the European health literacy survey (HLS- EU). Eur J Public Health. 2015;25:1053-8.

10. Hollander JE, Sites FD. The Transition from Reimagining to Recreating Health Care Is Now. NEJM Catalyst. 2020;8:0.

Manuscript received May 16, 2020; accepted for publication June 8, 2020.

Ruperto González-Pérez Allergy Department Hospital Universitario de Canarias 38320 Tenerife, Spain

E-mail: glezruperto@gmail.com 